\title{
RNA-guided diagnostics and therapeutics for next-generation individualized nanomedicine
}

\author{
Gennadi V. Glinsky
}

Sanford-Burnham Medical Research Institute, La Jolla, California, USA. Stanford University School of Medicine, Stanford, California, USA. Translational and Functional Genomics Laboratory, Genlight Technology Corp., La Jolla, California, USA.

\begin{abstract}
The absence of reliable quantitative laboratory tests for measurements of microRNAs and other classes of small noncoding RNAs in archived, formalin-fixed, paraffin-embedded human samples with sufficient specificity and sensitivity has significantly limited the development of clinically relevant noncoding RNA-based diagnostic and therapeutic applications. A report by Renwick et al. in this issue of the JCI presents a significant technical and methodological advance toward the development of reliable clinical laboratory-compatible multicolor RNA FISH methodology for molecular diagnostic applications and the near-term prospect of introduction of microRNA-based biomarkers into clinical practice. Further, this work is likely to advance the development of RNA-based therapeutics and next-generation individualized nanomedicine.
\end{abstract}

One of the significant uncertainties on the path toward the development and implementation of personalized, patientcentered therapeutic interventions is a lack of clarity in how to translate population-based knowledge of disease progression and treatment outcomes into clini$\mathrm{cal}$ and life-saving benefits for individual patients. Evolution of standard-of-care concepts for therapy of localized, earlystage prostate cancer is one of the best contemporary examples of this problem.

\section{Evolution of standard-of-care concepts for therapy of localized, early-stage prostate cancer}

Implementation of population-scale screening and nearly uniform use of aggressive therapies with definitive curative intent contributed to the reported 98\%-100\% five-year cancer-specific survival rates of patients diagnosed with prostate cancer in the United States since 1998 (SEER 13 areas statistics). Immediate curative intervention is the predominant therapy of choice, and 168,000 prostatectomies are performed in the United States each year to treat prostate cancer (1). However, there is a lack of consensus regarding the benefits of population-scale PSA

Conflict of interest: Gennadi V. Glinsky is the inventor on noncoding RNA-related patent applications and cofounder of the biotech start-up company Genlight Technology Corporation.

Citation for this article: J Clin Invest. 2013; 123(6):2350-2352. doi:10.1172/JCI69268 screening, and there is a perceived controversy about the potential for overdiagnosis and overtreatment of clinically insignificant disease that likely would not become life-threatening in a man's lifetime (2). High risk of complications significantly altering the quality of life of many patients undergoing immediate curative interventions, therapy failure, and disease relapse in a subgroup of patients represent other well-documented problems of the current approach to the clinical management of prostate cancer. Further, socioeconomic arguments describing significant overdiagnosis and unnecessary overtreatment have been presented in studies indicating that prevention of 1 prostate cancer death would require active treatment of 48 men for 9 years or 12 men for 14 years rary population-based cohorts reported cumulative 10-year prostate cancer-specific mortality in patients with low-risk disease of $2.4 \%$ and $0.7 \%$ in the surveillance group and curative intent therapy groups, respectively (5). These studies indicate that active surveillance may be a suitable treatment option for the majority of patients with low-risk, clinically indolent prostate cancer. Emerging clinical evidence indicates that active surveillance may be a safe, and even perhaps the preferred, option for older men diagnosed with a very low-grade or small-volume form of prostate cancer (6). Thus, for the patients with early-stage prostate cancer, active surveillance with $(3,4)$. Outcome studies from contempo- curative intent therapy, which entails the continuous effort of a multidisciplinary team of physicians, medical geneticists, and clinical pathologists to carefully document and monitor disease progression, seems to emerge as a consensus standard-of-care therapy choice.

\section{Noncoding RNAs and common human disorders}

Microarray and next-generation sequencing technologies dramatically altered our fundamental understanding of the expanding universe of the human transcriptome by revealing many thousands of long and small noncoding RNAs (lncRNAs and sncRNAs) that are intrinsically wired into high-complexity regulatory networks in human cells (7-10). Transcriptional outputs from genomic regions around the 22,000 protein-coding genes in the human genome generate an average of 10 transcripts each, including transcripts from sense, antisense, intronic, intergenic, pseudogenes, retrotransposons, and repetitive elements sequences. Emerging experimental and clinical evidence is mechanistically linking novel classes of sncRNAs and lncRNAs to a broad spectrum of common, polygenic human disorders (11-13). However, the lack of reliable quantitative labora tory tests for measurements of lncRNAs and sncRNAs in archived, formalin-fixed paraffin-embedded (FFPE) human samples with sufficient specificity and sensitivity has significantly limited the development of clinically relevant noncoding RNA-based translational applications. This is particularly relevant to prostate cancer which is often detected as a multifocal disease represented by several morphologically distinct microscopic tumors and requires careful examination of the biomarkers expression in individual cells within each individual lesion.

Novel RNA-based therapies broadly defined as therapeutic modalities aimed at the RNA targets and/or using nucleic acid chemistry-based therapeutic molecules 
(e.g., oligonucleotide drugs) are rapidly advancing into clinical trials (14-17). Notably, molecular targets for the successfully tested in the phase II (or above) clinical trials oligonucleotide-based therapeutics are outside of the so called "druggable" genome which is the primary target for development of small-molecule drugs (14). While most of these drugs are represented by the single-stranded second generation antisense oligonucleotides, there are small interfering RNAs (siRNAs) and the first microRNA-targeting drug, anti-miRNA122 oligonucleotide, Miravirsen, the locked nucleic acid-modified antisense oligonucleotide $(14,17)$.

\section{Research advances}

Last year, two papers reported credible clinical evidence documenting the efficient analytical performance of the lncRNA detection in FFPE samples $(18,19)$. In this issue of the JCI, Renwick et al. (20) report on a method for detection and measurement of microRNAs in clinical samples (20). This work represents a significant technical advance in the development of a reliable, clinical laboratory-compatible RNA FISH methodology for molecular diagnostic applications. Further, it provides compelling experimental evidence to support the feasibility of multicolor RNA FISH technology in clinical laboratories for highly accurate quantitative analyses of microRNAs and other classes of diseaseassociated sncRNAs such as trans-regulatory snpRNAs $(11,13)$. A notable limitation of the study is that the RNA FISH-based methodology was conclusively validated only for two tumor-specific miRNAs (miR205 and miR-375) in the small number of clinical samples analyzed to date. In the context of the expressed commitment of this group to explore the clinical utility of RNA FISH-based microRNA analyses in larger collections of clinical samples (20), this study has significant translational implications, because altered expression and activity of miR-205 and miR-375 has been linked to the increased likelihood of developing castration-resistant phenotype of human prostate cancer (13).

Recent experiments reveal that, similarly to lncRNAs, microRNAs can target polycomb repressive complexes to specific genomic loci, thereby contributing to the epigenetic reprogramming of chromatin and control of gene expression $(21,22)$. These findings, in combination with the development of a reliable molecular diag- nostic test for individualized target selection and validation, should accelerate the development of noncoding RNA-based therapeutics. Despite advances in our understanding of the microRNA contributions to disease-relevant genetic alterations, specific and efficient in vivo delivery of the RNA-based therapeutics and diagnostics remains a significant and largely intractable problem. Coincidently, several exciting reports in the nanobiotechnology and nanobioengineering fields suggest that the translation of noncoding RNA-based diagnostics and therapeutics into clinically beneficial applications is nearly at hand.

\section{Implications and future directions}

Advances in nanotechnology have been used to develop highly specific, inducible reagents for modulation of RNAs. Goldbased plasmonic nanomaterials and photothermal genetic engineering methods have been used to develop nanovectors that silence specific genes in vivo via photothermal activation of RNAi (23-25). A combination of photo-heating and near-infrared light has been used to target goldbased plasmonic nanoparticles and trigger release of gene-specific siRNAs and other classes of noncoding RNAs in a temporally and spatially defined manner (23-25). Ren et al. (26) reported the development and preclinical validation of a tumor-penetrating nanocomplex that combines a tandem tumor-penetrating and membrane-translocating peptide with siRNA, which was capable of specific and highly efficient in vivo delivery of siRNA deep into the tumor parenchyma (26).

DNA zipper and molecular tweezer technologies could also aid in the therapeutic manipulation of RNAs. Conceptually, a DNA zipper system consists of a normal strand (N) made up of conventional DNA bases, a weak strand (W) engineered to have inosine substituting for guanine, and an opening strand $(\mathrm{O})$ that contains a complementary sequence of conventional nucleotides. Incorporation of inosine in the W strand provides less than natural bonding affinities to $\mathrm{N}$ in forming the [N:W] helix (27). When the $\mathrm{O}$ strand sequence is introduced (a conventional base complement of $\mathrm{N}$ ), it competitively displaces $\mathrm{W}$ from the $[\mathrm{N}: \mathrm{W}]$ helix and forms an $[\mathrm{N}: \mathrm{O}]$ complex. Landon et al. (27) incorporated this principle in the development of a molecular nanodevice that can perform the functions of biological tweezers, which can sense, bind, and release target DNAs.
Conformational changes during the DNA zipper opening and closing cycles can be physically linked with fluorescent molecules to induce transitional states readily detectable by in vivo imaging techniques. We envision the design of a DNA zipper system to sense specific noncoding RNAs, which will function as an opening strand and trigger the release of therapeutic payloads and/or diagnostic imaging detectable states. These exciting new ideas articulate the basic principles for development of the next generation of diagnostic nanodevices and therapeutic nanovectors that implement gated access and control ondemand release (GACOR) nanotechnology. Development and preclinical evaluation of GACOR nanovectors for guided on-target delivery and on-demand controlled release of diagnostic and therapeutic agents will be crucial for the in vivo interrogation of functional significance and potential diagnostic and therapeutic utility of identified noncoding RNA targets as well as proteincoding transcripts.

In the near future, it should be possible to deliver oligonucleotide drugs with the single cell precision level in vivo. This highly anticipated technological breakthrough will be essential for critical testing of the validity of the cancer stem cell hypothesis and experimental and clinical assessment of the utility of selective therapeutic targeting of human tumor stemness pathways to prevent clinical progression (28). These exciting studies will facilitate the conclusive, evidence-based interrogation of the molecular and genetic mechanisms of disease states and enable unequivocal validation of diagnostic and therapeutic noncoding RNA targets.

\section{Acknowledgments}

I would like to thank my colleagues for critical comments and helpful discussions and suggestions during my work on this paper.

Address correspondence to: Gennadi V. Glinsky, Stanford University Medical School, Medical School Lab Surge Bldg., Room P214, 1201 Welch Road, Stanford, California 94305-5494, USA. Phone: 858.401.3470; Fax: 650.724.3229; E-mail: gglinsky@stanford.edu.

1. DeFrances CJ, Lucas CA, Buie VC, Golosinskiy A. 2006 National Hospital Discharge Survey. Natl Health Stat Report. 2008;(5):1-20.

2. Draisma G, et al. Lead times and overdetection due to prostate-specific antigen screening: estimates from the European Randomized Study of Screening for Prostate Cancer. J Natl Cancer Inst. 
2003;95(12):868-878

3. Schröder FH, et al. Screening and prostate-cancer mortality in a randomized european study. $N$ EnglJ Med. 2009;360(13):1320-1328.

4. Hugosson J, et al. Mortality results from the Göteborg randomised population-based prostate-cancer screening trial. Lancet Oncol. 2010;11(8):725-732.

5. Stattin P, et al. Outcomes in localized prostate cancer: National Prostate Cancer Register of Sweden follow-up study. J Natl Cancer Inst. 2010; 102(13):950-958

6. Tosoian JJ, et al. Active surveillance program for prostate cancer: an update of the Johns Hopkins experience. J Clin Oncol. 2011;29(16):2185-2190.

7. Rinn JL, Chang HY. Genome regulation by long noncoding RNAs. Annu Rev Biochem. 2012;81:145-166.

8. Glinsky GV. Phenotype-defining functions of multiple non-coding RNA pathways. Cell Cycle. 2008;7(11):1630-1639

9. Lee JT. Epigenetic regulation by long noncoding RNAs. Science. 2012;338(6113):1435-1439.

10. Wang KC, Chang HY. Molecular mechanisms of long noncoding RNAs. Mol Cell. 2011;43(6):904-914.

11. Glinskii $A B$, et al. Identification of intergenic trans-regulatory RNAs containing a disease-linked SNP sequence and targeting cell cycle progression/ differentiation pathways in multiple common human disorders. Cell Cycle. 2009;8(23):3925-3942.

12. Martin L, Chang HY. Uncovering the role of genomic "dark matter" in human disease. J Clin
Invest. 2012;122(5):1589-1595.

13. Glinskii $A B$, et al. Networks of intergenic longrange enhancers and snpRNAs drive castrationresistant phenotype of prostate cancer and contribute to pathogenesis of multiple common human disorders. Cell Cycle. 2011;10(20):3571-3597.

14. Lightfoot HL, Hall J. Target mRNA inhibition by oligonucleotide drugs in man. Nucleic Acids Res. 2012;40(21):10585-10595.

15. Olsen AL, Davies JM, Medley L, Breen D, Talbot DC, McHugh PJ. Quantitative analysis of survivin protein expression and its therapeutic depletion by an antisense oligonucleotide in human lung tumors. Mol Ther Nucleic Acids. 2012;1:e30.

16. Callies $\mathrm{S}$, et al. Integrated analysis of preclinical data to support the design of the first in man study of LY2181308, a second generation antisense oligonucleotide. Br JClin Pharmacol. 2011;71(3):416-428.

17. Lindow M, Kauppinen S. Discovering the first microRNA-targeted drug. I Cell Biol. 2012; 199(3):407-412.

18. Chisholm KM, Wan Y, Li R, Montgomery KD, Chang HY, West RB. Detection of long non-coding RNA in archival tissue: correlation with polycomb protein expression in primary and metastatic breast carcinoma. PLoS One. 2012;7(10):e47998.

19. Brunner AL, et al. Transcriptional profiling of IncRNAs and novel transcribed regions across a diverse panel of archived human cancers. Genome Biol. 2012;13(8):R75.
20. Renwick N, et al. Multicolor microRNA FISH effectively differentiates tumor types. J Clin Invest. 2013;123(6):2694-2702

21. Zardo G, et al. Polycombs and microRNA-223 regulate human granulopoiesis by transcriptional control of target gene expression. Blood. 2012;119(17):4034-4046

22. Zardo G, et al. Transcriptional targeting by microRNA-polycomb complexes: a novel route in cell fate determination. Cell Cycle. 2012;11(19):3543-3549.

23. Braun GB, et al. Laser-activated gene silencing via Gold NanoshellsiRNA conjugates. ACS Nano. 2009;3(7):2007-2015

24. Lu W, et al. Tumor site-specific silencing of NF-kappaB p65 by targeted hollow gold nanosphere-mediated photothermal transfection. Cancer Res. 2010;70(8):3177-3188.

25 . Lee SE, et al. Photonic gene circuits by optically addressable siRNA-Au nanoantennas. ACS Nano. 2012;6(9):7770-7780.

26. Ren Y, et al. Targeted tumor-penetrating siRNA nanocomplexes for credentialing the ovarian cancer oncogene ID4. Sci Transl Med.2012;4(147):147ra112.

27. Landon PB, Ramachandran S, Gillman A, Gidron T, Yoon D, Lal R. DNA zipper-based tweezers. Langmuir. 2012;28(1):534-540.

28. Glinsky GV, Berezovska O, Glinskii AB. Microarray analysis identifies a death-from-cancer signature predicting therapy failure in patients with multiple types of cancer.J Clin Invest. 2005;115(6):1503-1521.

\title{
Giardia: both a harmless commensal and a devastating pathogen
}

\author{
Herbert L. DuPont \\ University of Texas School of Public Health and Medical School, St. Luke's Episcopal Hospital, Baylor College of Medicine, \\ and the Kelsey Research Foundation, Houston, Texas, USA
}

\begin{abstract}
The highly prevalent protozoan Giardia lamblia is an enteropathogen that can be asymptomatic in some individuals, while leading to persistent diarrhea and substantial morbidity in others. In this issue of the JCI, Bartelt et al. describe a mouse model of the disease and investigate the contribution of coincident malnutrition with the development of symptomatic infection. This work in part explains how Giardia infection can lead to growth retardation, and may offer insights that guide future therapeutic strategies.
\end{abstract}

Giardia lamblia (synonymous with G. intestinalis and G. duodenalis), referred to herein as Giardia, was first detected in 1681 by Antonie van Leeuwenhoek when looking at his own stools and was later described in 1859 by Lambl (1). Finding the organism as frequently in patients without symptoms as in those with diarrheal illness has led

Conflict of interest: In the past 12 months, the author's university, University of Texas-Houston, has received research grants from Dr. Falk Pharma and INC (Santarus Corporation). The author has received compensation for serving as an expert witness for several lawsuits related to foodborne illness.

Citation for this article: J Clin Invest. 2013 123(6):2352-2354. doi:10.1172/JCI69932. many over the years to conclude that the organism is not a pathogen. Giardia can be identified in stools of $2 \%$ to $5 \%$ of presumably healthy people living in industrialized countries like the United States and in 20\% to $30 \%$ of people in developing regions (2). It is found in water sources and infects many animal species. The organism can be classified into at least 8 different genotypes called assemblages in humans and animals, with assemblages $\mathrm{A}$ and $\mathrm{B}$ being the most important in human infection. Each year in the United States, we identify approximately 20,000 people with Giardia infection, but the actual prevalence is estimated to be much higher.

\section{The two faces of Giardia infection}

In rural areas of the developing world, Giardia is ubiquitous and infects nearly all children, although most remain free of symptoms (3). In these endemic areas, infants experience an acute clinical disease only when first exposed to the protozoan, but quickly recover from infection without adverse long-term effects (4). Self-limiting diarrhea from a Giardia infection is common in young children newly attending day care centers $(5,6)$ and in international travelers (7) to endemic areas when first exposed to the protozoa. After initial exposure in otherwise healthy people, symptomatic infection occurs rarely. Risk factors for first symptomatic infection in young children were shown in one study carried out in rural Egypt to include young age, poverty, low education level, in-home storage of drinking water, and unhygienic treatment of girls related to gender discrimination (8). A proportion of infected people, mainly underweight children with preexistent mal- 